\title{
Combination Therapy With Tofacitinib Plus Intensive Granulocyte and Monocyte Adsorptive Apheresis as Induction Therapy for Refractory Ulcerative Colitis
}

\author{
Satoshi Tanida ${ }^{a}$,, Keiji Ozekia, Tsutomu Mizoshita ${ }^{a}$, Mika Kitagawa ${ }^{\text {a }}$, \\ Takanori Ozekia ${ }^{a}$, Mamoru Tanaka ${ }^{a}$, Hirotada Nishie ${ }^{a}$, Takaya Shimura ${ }^{a}$, \\ Eiji Kubota $^{\text {a }}$, Hiromi Kataoka ${ }^{a}$
}

\begin{abstract}
Background: The use of monotherapy with intensive granulocyte and monocyte adsorptive apheresis (GMA) or a Janus kinase (JAK) inhibitor has been limited to patients with refractory ulcerative colitis (UC). The efficacy and safety of combination therapy with tofacitinib (TOF) plus intensive GMA (two sessions per week) for refractory UC have not been evaluated.
\end{abstract}

Methods: This retrospective study evaluated the 10-week efficacy of combination therapy with TOF plus intensive GMA in patients with refractory UC.

Results: Of seven patients who received a combination therapy with TOF plus intensive GMA, 71.4\% achieved clinical remission at 10 weeks. The percentages of patients with mucosal healing and complete mucosal healing at 10 weeks were $100 \%$ and $42.9 \%$, respectively. The mean full Mayo score and endoscopic subscore at baseline were $8.71 \pm 0.80$ and $2.4 \pm 0.2$, respectively, and the corresponding values at 10 weeks were $1.57 \pm 0.48$ and $0.6 \pm 0.2(\mathrm{P}<0.01)$, respectively. Adverse events of an orolabial herpes and temporary increase in creatinine phosphokinase (CK) and triglyceride were observed in three patients.

Conclusions: Based on these outcomes, combination therapy with TOF plus intensive GMA was well tolerated and may be useful for induction of clinical remission in patients with refractory UC.

Keywords: Ulcerative colitis; Clinical remission; Mucosal healing

Manuscript submitted November 14, 2019, accepted December 9, 2019

${ }^{a}$ Department of Gastroenterology and Metabolism, Nagoya City University Graduate School of Medical Sciences, Nagoya City, Aichi Prefecture, Japan

${ }^{\mathrm{b}}$ Corresponding Author: Satoshi Tanida, Department of Gastroenterology and Metabolism, Nagoya City University Graduate School of Medical Sciences, 1 Kawasumi, Mizuho-cho, Mizuho-ku, Nagoya, Aichi 467-8601, Japan.

Email: stanida@med.nagoya-cu.ac.jp

doi: https://doi.org/10.14740/jocmr4037

\section{Introduction}

Ulcerative colitis (UC) is characterized by mucosal ulceration, rectal bleeding, diarrhea and abdominal pain. Current drug treatments for UC include aminosalicylic acid (5-ASA), corticosteroids, azathioprine and/or antagonists against tumor necrosis factor (TNF)- $\alpha$ or integrin $\alpha 4 \beta 7$ [1-5]. Studies have demonstrated the efficacy and safety of tofacitinib (TOF), which is a pan-Janus kinase (JAK) inhibitor, for the treatment of moderate to severe UC patients who failed to achieve clinical remission, did not respond to conventional medications, or lost response to TNF- $\alpha$ antagonists [6]. However, the reported efficacy rates of TOF monotherapy in inducing clinical remission in randomized patients with refractory UC were $18.5 \%$ in the Oral Clinical Trials for Tofacitinib in Ulcerative Colitis (OCTAVE) induction 1 trial and $16.6 \%$ in the OCTAVE induction 2 trial at 8 weeks [6]. Therefore, the use of TOF monotherapy has been limited when higher induction remission rates are the goal.

On the other hand, granulocyte and monocyte adsorptive apheresis (GMA) with Adacolumn ${ }^{\circledR}$ (JIMRO, Takasaki, Japan) is another effective and safe therapeutic option for patients with mild to moderate UC refractory to pharmacological therapy [7]. Furthermore, intensive GMA of two sessions per week has been recently shown to be superior to routine weekly GMA, both in remission rate and time to remission in patients with refractory UC [8]. However, the efficacy of intensive GMA on induction of clinical remission in patients with severe UC has not been satisfactory [9]. In this study, we retrospectively assessed the 10-week efficacy of a combination therapy with TOF and intensive GMA on patients with refractory UC.

\section{Patients and Methods}

\section{Patients}

Between December 2018 and November 2019, seven consecutive patients with moderate and severe UC were recruited from Nagoya City University Hospital and Midori Municipal Hospital for the study. Patients with UC who received a com- 
bination therapy with TOF and intensive GMA were enrolled in this study, according to the following criteria: 1) refractory or dependent on corticosteroids or loss of response to TNF- $\alpha$ antagonists; and 2) moderate to severe UC, with a Mayo score of 6 to 12 at baseline, including an endoscopic subscore of 2 to 3 , despite concurrent treatment with corticosteroids and/or TNF- $\alpha$ antagonists and 5-ASA. Only the dosages of corticosteroids were tapered off as appropriate. TOF was administered at $10 \mathrm{mg}$ twice a day for 8 weeks as induction therapy and, subsequently, $5 \mathrm{mg}$ twice a day, and intensive GMA was administered in two sessions a week for a total of 10 times. The study protocol was approved by Nagoya City University ethics committee as a representative of both the hospitals (\#6019-0164), and written informed consent was obtained from all patients prior to enrollment.

\section{Treatment and assessments}

The primary outcome was the 10 -week clinical remission rate of the combination therapy with TOF and intensive GMA, and the secondary outcomes were the rates of clinical response and mucosal healing at 10 weeks. Disease activity and severity were assessed using the full Mayo score [2] at baseline and at 10 weeks. Mucosal healing was defined as a Mayo endoscopy subscore of either 0 or 1 , and complete mucosal healing was defined as a subscore of 0 . Clinical remission was defined as a Mayo score of $\leq 2$ with no individual subscore of $>1$. Any adverse event, including the date of onset, severity, outcome and relationship of events with the therapy, was recorded.

\section{Statistical methods}

The data were presented as means \pm standard error of the mean, and comparisons were made by using paired $t$-test. Statistical analyses were performed by SPSS V24 software. A significant level of 0.01 was used for all statistical tests, and two-tailed tests were applied as appropriate.

\section{Results}

The demographic data are shown in Table 1. The mean age was 53.3 years, and the mean disease duration was 16.1 years. In terms of colonic involvement, three had extensive colitis and four had left-sided colitis. The concurrent medications included 5 -ASA, corticosteroids and TNF- $\alpha$ antagonists. Of seven patients, two were corticosteroid-refractory, five were corticosteroid-dependent and four experienced loss of response to TNF- $\alpha$ antagonists. The mean full Mayo score and C-reactive protein (CRP) levels at baseline were 8.71 and $0.71 \mathrm{mg} / \mathrm{dL}$ (Table 1).

Among the seven patients who were given a combination therapy of TOF and intensive GMA, the mean full Mayo scores decreased from $8.71 \pm 0.80$ at baseline to $1.57 \pm 0.48$ at 10 weeks, and mean endoscopic subscore decreased from $2.4 \pm$ 0.2 at baseline to $0.6 \pm 0.2$ at 10 weeks (Fig. 1a, b). These improvements were significant $(\mathrm{P}<0.01)$. On the other hand, the
Table 1. Baseline Demographic Data of Seven Cases That Received Combination Therapy With TOF and Intensive GMA for Refractory UC

\begin{tabular}{ll}
\hline Demography & \\
\hline Male/female (N) & $2 / 5$ \\
Age (years) & 53.3 \\
Mean disease duration (years) & 16.1 \\
Location of lesions (N) & 3 \\
$\quad$ Extensive & 4 \\
$\quad$ Left-sided & \\
Severity (N) & $6 / 1$ \\
$\quad$ Moderate/severe & \\
Concomitant medications for UC (N) & 6 \\
$\quad$ Corticosteroids/budesonide & 7 \\
$\quad$ Mesalamine & 1 \\
$\quad$ Azathioprine & 4 \\
\hline Prior use of anti-TNF- $\alpha$ antibodies (N) & 8.71 \\
Full Mayo score at baseline & 2.4 \\
Endoscopic sub-score at baseline & 0.71 \\
\hline CRP at baseline (mg/dL) &
\end{tabular}

The data are presented as means. TOF: tofacitinib; GMA: granulocyte and monocyte adsorptive apheresis; UC: ulcerative colitis; TNF: tumor necrosis factor; CRP: C-reactive protein.

mean CRP level decreased from $0.71 \pm 0.49 \mathrm{mg} / \mathrm{dL}$ at baseline to $0.02 \pm 0.005 \mathrm{mg} / \mathrm{dL}$ at 10 weeks with no significant difference $(P=0.20)$ after the combination therapy with TOF and intensive GMA (Fig. 1c). The rate of clinical remission at 10 weeks was $71.4 \%$. At 10 weeks, mucosal healing was observed in all cases (100\%) and complete mucosal healing [10] was seen in three cases $(42.9 \%)$. In addition, all six patients who received oral prednisolone or rectal budesonide were able to withdraw corticosteroids at 10 weeks $(100 \%)$ (Table 2).

\section{Safety}

Adverse events were observed in three patients: one had orolabial herpes, one had transient increase in creatinine phosphokinase due to intense physical exercise and one had triglyceride increase. In these cases, TOF was not discontinued and 10 sessions of intensive GMA were completed. In general, combination therapy with TOF and intensive GMA was safe and well tolerated.

\section{Discussion}

In this study, we reported the efficacy of a combination of TOF induction and intensive GMA in seven consecutive cases of refractory UC.

TOF is a small oral molecule and is a pan-JAK inhibitor that suppresses the JAK signal transducers and the activators 

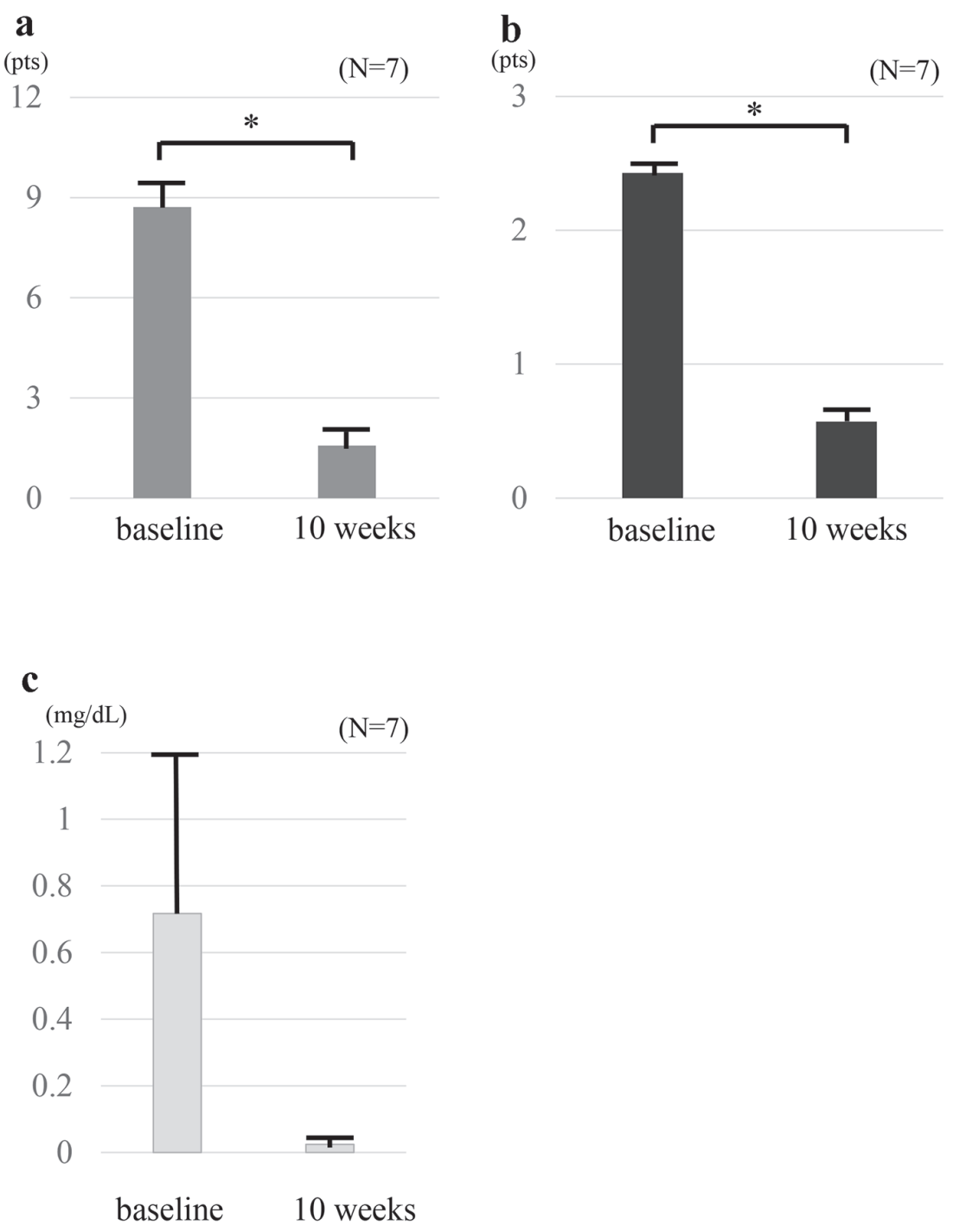

Figure 1. Clinical outcomes at baseline and at 10 weeks in seven patients who received combination therapy TOF and intensive GMA. The mean full Mayo scores (a) and endoscopic sub-scores (b) show significant differences between at baseline and 10 weeks $(P<0.01)$. The mean full Mayo scores decreased from $8.71 \pm 0.80$ at baseline to $1.57 \pm 0.48$ at 10 weeks, and the mean endoscopic subscore decreased from $2.4 \pm 0.2$ at baseline to $0.6 \pm 0.2$ at 10 weeks. CRP values (c) show no significant difference between at baseline and 10 weeks $(P=0.20)$. The mean CRP level decreased from $0.71 \pm 0.49 \mathrm{mg} / \mathrm{dL}$ at baseline to 0.02 $\pm 0.005 \mathrm{mg} / \mathrm{dL}$ at 10 weeks. The values are presented as means \pm standard error values and were made by using paired $t$-test. ${ }^{*} \mathrm{P}<0.01$. TOF: tofacitinib; GMA: granulocyte and monocyte adsorptive apheresis; CRP: C-reactive protein.

of transcription pathway, thereby down-regulating the expressions of multiple immune-relevant mediators and inflammatory cytokines that are implicated in the pathogenesis of inflammatory bowel disease (IBD) $[11,12]$. In the clinical setting, TOF has been an efficacious therapy to induce clinical remission in patients with moderate to severe active $\mathrm{UC}[6,13]$. Two phase 3 trials that investigated TOF as induction therapy, the OCTAVE induction 1 and 2, which were double-blind placebo-controlled phase 3 trials that investigated TOF as induction therapy, revealed that the overall clinical remission rate at week 8 was $18.5 \%$ in patients who were randomized to TOF at a dose of $10 \mathrm{mg}$ twice a day; and $8.2 \%$ in patients allocated to placebo $(\mathrm{P}=0.007)$ in the OCTAVE induction 1 trial; $16.6 \%$ in patients who were randomized to TOF at a dose of $10 \mathrm{mg}$ twice a day; and $3.6 \%$ in patients allocated to placebo $(\mathrm{P}<$ $0.001)$ in the OCTAVE induction 2 trial. Therefore, TOF monotherapy for refractory UC has limitation, and additional treatment would be necessary for active UC patients who are biologically naive and have lost response to TNF- $\alpha$ antagonists. In the present study on seven patients who received combination therapy with TOF plus intensive GMA, the rate of clinical remission at 10 weeks was $71.4 \%$. Based on this outcome, the addition of intensive GMA to TOF monotherapy appeared to be an effective therapy that induced clinical remission.

In Europe and Japan, GMA is available for the treatment of patients with active IBD that has become refrac- 


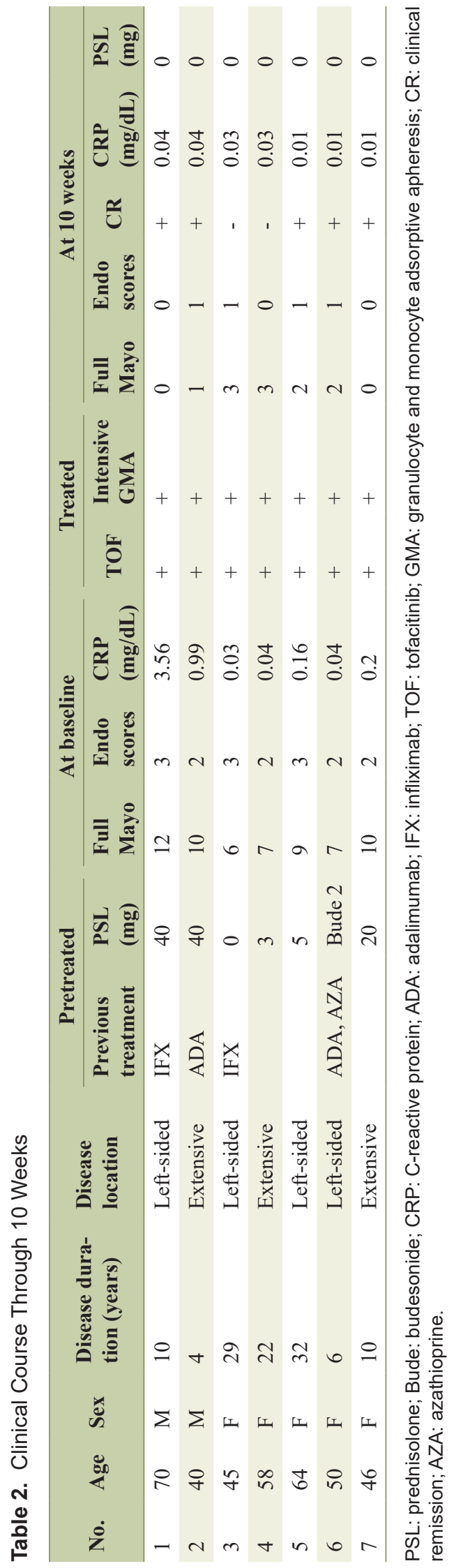

tory to standard drug-based medications, including TNF- $\alpha$ blockers. GMA depletes the elevated and activated myeloid lineage of leukocytes and has been associated with marked downregulation of inflammatory cytokines, including interleukin (IL)-1 $\beta$, IL-6, IL-8 and TNF- $\alpha$, which are released by myeloid leukocytes and lymphocytes, most likely through an upstream mechanism that involves the adsorption of cytokineproducing cells $[14,15]$. Furthermore, in patients with active $\mathrm{UC}$, downregulation of the leukocyte adhesion molecule Lselectin and neutrophil adhesion to endothelial cells activated by IL- $1 \beta$ were observed after in vitro exposure of peripheral blood to G-1 beads that composed a main part of Adacolumn [16]. These suggested that the combination therapy worked by drastically downregulating the circulating inflammatory cytokines and the expressions of adhesive molecules on the activated granulocytes, which was an effect of GMA, and by downregulating the local inflammatory cytokines at the microenvironmental sites in the gut mucosa, which was an effect of TOF, thereby, inducing rapid clinical remission $[17,18]$. In addition, serious adverse side effects have been rare in patients receiving GMA $[9,19]$.

Endoscopic evaluation of patients who received TOF for refractory UC showed significant $(\mathrm{P}<0.001) 8$-week mucosal healing rates of $31.3 \%$ (vs. $15.6 \%$ in the placebo) in the OCTAVE induction 1 trial and $28.4 \%$ (vs. $11.6 \%$ in the placebo) in the OCTAVE induction 2 trial. In the present study on seven patients who received a combination therapy of TOF and intensive GMA, the rate of mucosal healing at 10 weeks was $100 \%$ and that of complete mucosal healing rate was $42.9 \%$. The mean endoscopic subscore was significantly lower at 10 weeks than at baseline. Based on these outcomes, addition of intensive GMA to TOF monotherapy appeared to be effective in inducing mucosal healing $[17,18]$. An additional study on a large cohort of patients is warranted to verify this finding.

Increased risks for hematologic disorders; opportunistic infections, including Herpes zoster; and elevation in CK or lipid levels have been reported with the use of TOF [6]. In the present study, orolabial herpes and increase in CK and triglycerides were seen in three cases, respectively. These were consistent with the adverse events reported in the OCTAVE induction 1 and 2 trials. The present study has limitations on sample size and exposure time to fully characterize the safety of combination therapy of TOF and intensive GMA.

In conclusion, combination therapy with TOF and intensive GMA was well tolerated and may be useful for the induction of clinical remission and mucosal healing in patients with refractory UC.

\section{Acknowledgments}

None to declare.

\section{Financial Disclosure}

None to declare. 


\section{Conflict of Interest}

The authors declare that there is no conflict of interest regarding the publication of this paper.

\section{Informed Consent}

Written informed consent was obtained from all patients.

\section{Author Contributions}

ST contributed to data acquisition and manuscript preparation. KO, TM, MK, TO, MT, HN, TS, EK and HK contributed to data acquisition and review of the manuscript.

\section{Abbreviations}

GMA: granulocyte and monocyte adsorptive apheresis; JAK: Janus kinase; UC: ulcerative colitis; TOF: tofacitinib; CK: creatinine phosphokinase; 5-ASA: 5-aminosalicylic acid; OCTAVE: Oral Clinical Trials for Tofacitinib in Ulcerative Colitis; CRP: C-reactive protein

\section{References}

1. Ordas I, Eckmann L, Talamini M, Baumgart DC, Sandborn WJ. Ulcerative colitis. Lancet. 2012;380(9853):16061619 .

2. Schroeder KW, Tremaine WJ, Ilstrup DM. Coated oral 5 -aminosalicylic acid therapy for mildly to moderately active ulcerative colitis. A randomized study. N Engl J Med. 1987;317(26):1625-1629.

3. Rutgeerts P, Sandborn WJ, Feagan BG, Reinisch W, Olson A, Johanns J, Travers S, et al. Infliximab for induction and maintenance therapy for ulcerative colitis. N Engl J Med. 2005;353(23):2462-2476.

4. Sandborn WJ, van Assche G, Reinisch W, Colombel JF, D'Haens G, Wolf DC, Kron M, et al. Adalimumab induces and maintains clinical remission in patients with moderate-to-severe ulcerative colitis. Gastroenterology. 2012;142(2):257-265 e251-253.

5. Feagan BG, Rutgeerts P, Sands BE, Hanauer S, Colombel JF, Sandborn WJ, Van Assche G, et al. Vedolizumab as induction and maintenance therapy for ulcerative colitis. N Engl J Med. 2013;369(8):699-710.

6. Sandborn WJ, Su C, Sands BE, D'Haens GR, Vermeire S, Schreiber S, Danese S, et al. Tofacitinib as induction and maintenance therapy for ulcerative colitis. N Engl J Med. 2017;376(18):1723-1736.

7. Ljung T, Thomsen OO, Vatn M, Karlen P, Karlsen LN, Tysk C, Nilsson SU, et al. Granulocyte, monocyte/macrophage apheresis for inflammatory bowel disease: the first 100 patients treated in Scandinavia. Scand J Gastroenterol. 2007;42(2):221-227.
8. Sakuraba A, Motoya S, Watanabe K, Nishishita M, Kanke K, Matsui T, Suzuki Y, et al. An open-label prospective randomized multicenter study shows very rapid remission of ulcerative colitis by intensive granulocyte and monocyte adsorptive apheresis as compared with routine weekly treatment. Am J Gastroenterol. 2009;104(12):29902995.

9. Hanai H, Watanabe F, Yamada M, Sato Y, Takeuchi K, Iida T, Tozawa $\mathrm{K}$, et al. Adsorptive granulocyte and monocyte apheresis versus prednisolone in patients with corticosteroid-dependent moderately severe ulcerative colitis. Digestion. 2004;70(1):36-44.

10. Reinisch W, Sandborn WJ, Panaccione R, Huang B, Pollack PF, Lazar A, Thakkar RB. 52-week efficacy of adalimumab in patients with moderately to severely active ulcerative colitis who failed corticosteroids and/or immunosuppressants. Inflamm Bowel Dis. 2013;19(8):17001709.

11. Lovato P, Brender C, Agnholt J, Kelsen J, Kaltoft K, Svejgaard A, Eriksen KW, et al. Constitutive STAT3 activation in intestinal $\mathrm{T}$ cells from patients with Crohn's disease. J Biol Chem. 2003;278(19):16777-16781.

12. Schreiber S, Rosenstiel P, Hampe J, Nikolaus S, Groessner B, Schottelius A, Kuhbacher T, et al. Activation of signal transducer and activator of transcription (STAT) 1 in human chronic inflammatory bowel disease. Gut. 2002;51(3):379-385.

13. Sandborn WJ, Ghosh S, Panes J, Vranic I, Su C, Rousell $\mathrm{S}$, Niezychowski W, et al. Tofacitinib, an oral Janus kinase inhibitor, in active ulcerative colitis. N Engl J Med. 2012;367(7):616-624.

14. Kashiwagi N, Hirata I, Kasukawa R. A role for granulocyte and monocyte apheresis in the treatment of rheumatoid arthritis. Ther Apher. 1998;2(2):134-141.

15. Saniabadi AR, Hanai H, Takeuchi K, Umemura K, Nakashima M, Adachi T, Shima C, et al. Adacolumn, an adsorptive carrier based granulocyte and monocyte apheresis device for the treatment of inflammatory and refractory diseases associated with leukocytes. Ther Apher Dial. 2003;7(1):48-59.

16. Kashiwagi N, Sugimura $\mathrm{K}$, Koiwai $\mathrm{H}$, Yamamoto $\mathrm{H}$, Yoshikawa T, Saniabadi AR, Adachi M, et al. Immunomodulatory effects of granulocyte and monocyte adsorption apheresis as a treatment for patients with ulcerative colitis. Dig Dis Sci. 2002;47(6):1334-1341.

17. Tanida S, Mizoshita T, Nishie H, Ozeki K, Katano T, Kubota E, Kataoka H, et al. Combination therapy with adalimumab plus intensive granulocyte and monocyte adsorptive apheresis in patients with refractory ulcerative colitis. J Clin Med Res. 2015;7(11):884-889.

18. Tanida S, Mizoshita T, Ozeki K, Katano T, Tanaka M, Nishie $\mathrm{H}$, Shimura $\mathrm{T}$, et al. Combination therapy with intensive granulocyte and monocyte adsorptive apheresis plus ustekinumab in patients with refractory Crohn's disease. Ther Apher Dial. 2018;22(3):295-300.

19. Thanaraj S, Hamlin PJ, Ford AC. Systematic review: granulocyte/monocyte adsorptive apheresis for ulcerative colitis. Aliment Pharmacol Ther. 2010;32(11-12):12971306. 